\title{
Avaliação da preceptoria na residência médica em cirurgia geral, no centro cirúrgico, comparação entre um hospital universitário e um hospital não universitário
}

\author{
Assessment of preceptorship in general surgery residency in the operating \\ room, comparison between a teaching hospital and a non teaching hospital
}

Elizabeth Gomes Santos, TCBC-RJ'; Rafael Rodriguez Ferreira, TCBC-RJ2; Vera Lúcia Mannarino33;

Elizabeth Menezes Teixeira lehere; ${ }^{4}$ Rosane S. Goldwasser ${ }^{5}$; Gullherme Pinto Bravo Neto, TCBC-RJ

\section{R E S U M O}

\begin{abstract}
Objetivo: Avaliar e comparar a preceptoria no programa de residência médica em Cirurgia Geral, no centro cirúrgico, em um hospital universitário e em um hospital não universitário, a partir da ótica dos residentes que ingressaram em 2010 e 2011. Métodos: Questionário aplicado aos residentes, modificado de Sarker SK, Vincent C, e Darzi AW e usando-se a escala de Likert para qualificar o ítem pesquisado sobre as atitudes dos preceptores. A comparação da distribuição das respostas entre os dois hospitais foi analisada pelo teste de $\chi^{2}$ para tendências. Resultados: No hospital universitário foram avaliados 12 preceptores por sete residentes. No hospital não universitário foram 11 preceptores avaliados por 13 residentes. O hospital não universitário apresentou a tendência de resposta discordante e indiferente (DC, D e I) maior que o hospital universitário. Só o resultado de uma pergunta apresentou significância estatística. Não houve diferença significativa na comparação das respostas nas demais perguntas entre os dois hospitais. Conclusão: Os hospitais apresentaram preceptoria semelhante.
\end{abstract}

Descritores: Avaliação. Educação profissionalizante. Internato e Residência. Tutoria. Cirurgia Geral.

\section{INTRODUÇÃO}

$\mathrm{N}^{2}$ história da educação médica é frequentemente citada a figura de um profissional mais experiente que auxilia na formação dos aprendizes e que é chamado "preceptor"1,2.

A aprendizagem não se faz sozinha. Na opinião de Vygotsky "a aprendizagem é concebida do exterior do sujeito para o interior e é fruto de suas interações com o mundo"3,4. Assim sendo, o preceptor tem a função de propiciar situações para que o aprendiz possa construir seu conhecimento. Sua principal função é agir como facilitador da aquisição do conhecimento, estimulando o aprendiz a fazer suas próprias descobertas ${ }^{5}$. É papel do preceptor fazer de seu aluno um médico curioso que pensa, questiona e chega às suas próprias conclusões, não sendo apenas um receptor de informações. Sua experiência deve ser levada em consideração. Ele deve ser reconhecido como aquele que tem mais experiência, mas não deve ser visto como dono absoluto do conhecimento.

Botti $^{2}$ diz que na literatura médica encontram-se citadas diferentes atribuições para o preceptor, sendo as essenciais: orientar, dar suporte, ensinar e compartilhar experiências que ajudem o aprendiz a adaptar-se ao exercício de uma profissão que vive em constante mudança. Ampliando-se um pouco os conceitos de Perrenoud 6 sobre o papel da escola, extendendo-os para os preceptores, podemos dizer que também é papel dos preceptores oferecer aos aprendizes as ferramentas necessárias para dominar a vida e compreender o mundo.

A Residência Médica (RM) é caracterizada por treinamento em serviço. É o cenário onde os processos de ensino-aprendizagem e o de assistência precisam estar em equilíbrio, portanto tão importante quanto o desenvolvimento de habilidades técnicas é o desenvolvimento da prática do comportamento responsável, moldado pela ética e pelo espírito de cooperação que devem nortear a prática médica. Um bom programa de residência, e neste caso em Cirurgia Geral (CG), visa proporcionar uma formação ampla para que, ao término, o futuro cirurgião esteja qualificado ao exercício de sua especialidade como um profissional capaz de oferecer à sociedade um atendimento com o máximo de qualidade e de forma humanizada, desenvolvendo habilidades no domínio afetivo, como aprimoramento de juízos e preceitos morais, requisitos necessários para sua inserção no mercado de trabalho, com total auto-

Trabalho realizado no Hospital Universitário Clementino Fraga Filho (HUCFF-UFRJ) e no Hospital Municipal Salgado Filho (HMSF).

1. Mestre em Cirurgia Geral - UFRJ; 2. Cirurgião do Hospital Municipal Salgado Filho - SMS-RJ; 3. Mestre em Medicina - UFRJ; 4. Doutora em Educação, Pesquisadora do NUTES/UFRJ; 5. Aluna do Curso de Doutorado, UFRJ; 6. Professor Adjunto FM-UFRJ. 
nomia. No momento atual, no qual a globalização promove uma grande circulação de informações e, em paralelo, há um grande desenvolvimento técnológico, é preciso que o programa privilegie o desenvolvimento de diversas competências, a fim de que haja integração, na prática, de todos os saberes ${ }^{7}$.

A primeira turma de RM do Hospital Universitário Clementino Fraga Filho da Universidade Federal do Rio de Janeiro (HUCFF/UFRJ) teve início em meados de 1978, logo após a inauguração do hospital. Até 2011, os residentes têm sido avaliados ao final da residência, como preconiza o Conselho Nacional de Residência Médica (CNRM), principalmente no que diz respeito ao domínio cognitivo ${ }^{8}$. Não houve, até agora, uma avaliação da preceptoria em CG. Isto equivale a afirmar que em nenhum momento se conheceu o ponto de vista dos residentes em relação aos seus preceptores no principal cenário de prática da RMCG, o centro cirúrgico (CC).

Até a Década de 60, os programas de residência se concentravam nos hospitais públicos assistenciais e nos universitários. Com a criação, pelo Ministério da Saúde (MS), do Fator de Incentivo ao Desenvolvimento do Ensino e Pesquisa Universitários, que estabelece um adicional de pagamento às instituições que oferecem programas de ensino, a RM entre eles, houve uma grande difusão do credenciamento de programas de residência em hospitais não universitários, municipais e estaduais, não ligados à escolas médicas ${ }^{9}$. Não temos conhecimento de avaliação da preceptoria feita pelos residentes, nestes hospitais.

\section{MÉTODOS}

O Serviço de Cirurgia Geral do HUCFF/UFRJ conta com dois programas de residência médica em Cirurgia Geral credenciados: Cirurgia Geral e Cirurgia Geral - Programa Avançado. O HNU é credenciado somente para CG. A área de interesse do estudo foi os preceptores do Serviço de CG dos dois serviços. No HU há servidores técnicoadministrativos (médicos) e docentes da UFRJ, todos com a mesma função: preceptores. No Hospital Não Universitário (HNU) não há nenhum preceptor que seja também docente formal em faculdade de medicina.

Foi aplicado um questionário de resposta fechada, aos residentes dos dois Serviços. Dentre os residentes do primeiro ano (R1) de CG do HU, apenas três participaram da pesquisa. Os outros três encontravam-se em rodízios por especialidades cirúrgicas e, até o momento da pesquisa, não haviam tido contato com os preceptores da CG. Os outros dois são R1 da CG - Programa Avançado. No HNU todos os residentes (R1, e R2 de CG) participaram da pesquisa. O questionário foi modificado de Sarker SK, Vincent $C$ e Darzi $A W^{10}$ e usou-se a escala de Likert' ${ }^{11}$ para qualificar o ítem pesquisado sobre as atitudes dos preceptores. O critério de inclusão dos preceptores foi: supervisão direta no CC por mais de seis meses. Um residente de cada hospital foi responsável pela codificação dos preceptores e esse código só foi conhecido pelos próprios residentes. Foi garantido o anonimato das respostas. Os seguintes itens foram avaliados: 1) Não deixa o residente continuar a operação ao cometer um pequeno erro; 2) Explica o procedimento pelo menos uma vez; 3) Orienta o residente assumindo a posição de cirurgião e não a de auxiliar; 4) Fica concentrado na operação; 5) Demonstra estar sempre com pressa; 6) Exerce pressão sobre o residente para terminar logo a operação; 7) Orienta a operação no campo; 8) Permanece na sala enquanto dura a operação; 9) Supervisiona de maneira agressiva; 10) Discute a evolução do residente e oferece sugestões.

As respostas foram distribuídas da seguinte maneira: a) discordo completamente, b) discordo, c) indiferente, d) concordo, e) concordo completamente.

Para o uso do questionário, partiu-se da premissa que os residentes são os melhores avaliadores das qualidades dos preceptores. Tais questionários são instrumentos efetivos de avaliação, embora passíveis de crítica pelo componente subjetivo e pelo pequeno número de avaliadores.

\section{RESULTADOS}

No hospital universitário 12 preceptores foram avaliados por sete residentes. Um dos residentes não respondeu o questionário. No hospital não universitário 11 preceptores foram avaliados por 13 residentes.

Foram excluídas, em ambos os hospitais, as avaliações respondidas com "indiferente" em todos os quesitos, por significar que, o residente que assim respondeu, não havia tido contato com aquele preceptor (pactuação prévia).

Foram obtidas 227 respostas válidas. A análise descritiva foi apresentada sob forma de tabela (Tabela 1) com os dados observados, expressos em percentual (\%).

A comparação da distribuição das respostas entre os dois hospitais foi analisada pelo teste de $\chi^{2}$ para tendências. O critério de determinação de significância adotado foi o nível de $5 \%$.

Foi observada uma tendência de descontentamento em relação à preceptoria no HNU por apresentar uma tendência de respostas discordante e indiferente (DC, $\mathrm{D} \mathrm{e} \mathrm{I)} \mathrm{maior} \mathrm{do} \mathrm{que} \mathrm{no} \mathrm{HU.}$

Só o resultado de um pergunta, a número quatro, apresentou significância estatística. Não existe diferença significativa, ao nível de 5\%, na distribuição das respostas nas demais perguntas entre os dois hospitais.

\section{DISCUSSÃO}

A distância que separa um bom preceptor daquele sem a necessária competência para ensinar é muito 
Tabela 1 - Distribuição relativa (\%) das respostas ao questionário segundo o hospital.

\begin{tabular}{|c|c|c|c|c|c|c|c|c|}
\hline \multirow{2}{*}{\multicolumn{2}{|c|}{ Pergunta }} & \multirow[t]{2}{*}{ Hospital } & \multicolumn{5}{|c|}{ Respostas } & \multirow[t]{2}{*}{$p$ valor ${ }^{a}$} \\
\hline & & & $D C$ & $\mathrm{D}$ & 1 & $\mathrm{C}$ & $\mathrm{CC}$ & \\
\hline \multirow[t]{2}{*}{ P1 } & \multicolumn{8}{|l|}{ Não deixa o residente } \\
\hline & cometer um pequeno erro & SF & 39,7 & 39,0 & 9,2 & 6,4 & 5,7 & \\
\hline \multirow[t]{2}{*}{ P2 } & Explica o procedimento & HUCFF & 3,1 & 10,8 & 12,3 & 63,1 & 10,8 & 0,22 \\
\hline & pelo menos uma vez & SF & 3,6 & 11,4 & 9,2 & 46,8 & 29,1 & \\
\hline \multirow[t]{3}{*}{ P3 } & Orienta o residente & & & & & & & \\
\hline & assumindo a posição de & HUCFF & 15,4 & 46,2 & 6,2 & 32,3 & 0,0 & 0,87 \\
\hline & cirurgião e não de auxiliar & SF & 26,2 & 34,8 & 9,9 & 18,4 & 10,6 & \\
\hline \multirow[t]{2}{*}{ P4 } & Fica concentrado na & HUCFF & 0,0 & 1,5 & 3,1 & 58,5 & 36,9 & 0,010 \\
\hline & operação & SF & 4,3 & 7,8 & 14,2 & 38,3 & 35,5 & \\
\hline \multirow[t]{2}{*}{ P5 } & Demonstra estar sempre & HUCFF & 30,8 & 40,0 & 9,2 & 15,4 & 4,6 & 0,16 \\
\hline & com pressa & SF & 25,5 & 35,5 & 12,8 & 16,3 & 9,9 & \\
\hline \multirow[t]{3}{*}{ P6 } & Exerce pressão sobre o & & & & & & & \\
\hline & residente para terminar & HUCFF & 30,8 & 41,5 & 6,2 & 18,5 & 3,1 & 0,11 \\
\hline & logo a operação & SF & 23,4 & 38,3 & 12,1 & 16,3 & 9,9 & \\
\hline \multirow[t]{2}{*}{ P7 } & Orienta a operação & HUCFF & 1,5 & 4,6 & 4,6 & 60,0 & 29,2 & 0,60 \\
\hline & no campo & SF & 5,7 & 6,4 & 6,4 & 42,6 & 39,0 & \\
\hline \multirow[t]{2}{*}{ P8 } & Permanece na sala & HUCFF & 3,1 & 15,4 & 12,3 & 44,6 & 24,6 & 0,57 \\
\hline & enquanto dura a operação & $\mathrm{O} \quad \mathrm{SF}$ & 9,9 & 14,2 & 9,9 & 36,2 & 29,8 & \\
\hline \multirow[t]{2}{*}{ P9 } & Supervisiona de maneira & HUCFF & 43,1 & 43,1 & 9,2 & 3,1 & 1,5 & 0,18 \\
\hline & agressiva & SF & 44,7 & 31,9 & 8,5 & 9,9 & 5,0 & \\
\hline \multirow[t]{3}{*}{ P10 } & Discute a evolução do & & & & & & & \\
\hline & residente e oferece & HUCFF & 4,6 & 16,9 & 23,1 & 38,5 & 16,9 & 0,30 \\
\hline & sugestões & SF & 5,0 & 11,4 & 23,4 & 36,2 & 24,1 & \\
\hline
\end{tabular}

a teste de $\chi 2$ para tendências.

Questionário modificado de Sudip K. Sarker, Charles Vincent e Ara W. Darzi.

Análise estatística realizada pela Professora Rosângela Noé (Bioestatística da Divisão de Pesquisa do HUCFF/UFRJ)

pequena ${ }^{12}$. Ser um excelente cirurgião não quer dizer ser, automaticamente, um bom preceptor. Ensinar e desenvolver habilidades técnicas é um desafio ${ }^{13}$. Esse aprendizado ocorre muitas vezes em meio a um ambiente de tensão, algumas vezes com pouca preparação, e, para que não haja prejuízo para o paciente, é preciso que ele se faça sempre de forma supervisionada por preceptores adequadamente treinados. Um bom preceptor faz com que o futuro profissional de Medicina consolide o aprendizado por meio da prática em serviço.

A residência médica é o mais importante cenário de aprendizado após a graduação. O conteúdo programático da RMCG, embora com pouca consistência, foi definido pela $\mathrm{CNRM}^{8}$. Não está estabelecido o que é necessário para o ensino e desenvolvimento das habilidades técnicas indispensáveis para a vida prática de todo cirurgião.

Há hoje uma grande preocupação em relação à preceptoria. Percebe-se, em todos os lugares, uma necessidade de capacitação dos profissionais que exercerão essa atividade ${ }^{14}$. Em várias universidades em todo o Brasil existe um movimento em favor da capacitação de preceptores.
Para o exercício é necessário que o profissional tenha conhecimentos dos quatro pilares (saberes) da educação: o saber - conhecer, o saber - fazer, o saber - ser e o saber - conviver, já que o preceptor atua como exemplo de conduta técnica e ética. É preciso que ele admita não ser o "dono" de todos os saberes e que esteja disposto a partilhar o processo de aprendizado com os aprendizes ${ }^{15}$.

Cirurgiões experientes e educadores afirmam ser de grande importância o ensino no CC, já que é lá onde os residentes passam grande parte de sua carga horária ${ }^{15}$, porém, mesmo nesse ambiente tão familiar a eles, aqueles que exercem a preceptoria não têm muito conhecimento sobre o seu papel e quais os objetivos a serem alcançados $^{14}$, e praticam, na maioria das vezes, uma preceptoria intuitiva.

Com base em periódicos e na legislação brasileira, Mills ${ }^{12}$ define preceptor como aquele profissional que não pertence necessariamente à universidade, mas, tem importante papel na inserção e socialização do recém-graduado no ambiente de trabalho. O preceptor existe, qualquer que seja a classificação do cuidado que o hospital em que trabalha oferece, porque a RM existe em hospitais 
universitários e em hospitais não universitários. A qualidade da preceptoria pode modificar o ganho de habilidades técnicas do futuro cirurgião, assim como o desenvolvimento de outras competências. É dever do preceptor identificar as habilidades dos residentes, treiná-los, sugerir mudanças e corrigir seus erros ${ }^{2}$. Para que essa transmissão de conhecimentos se faça, ele deve desenvolver uma atitude reflexiva e, através de auto-avaliações e das avaliações dos seus residentes, melhorar seu processo educativo.

A carreira docente formal não é exigida para o exercício da preceptoria na RM; não há uma definição dos requisitos necessários nem avaliação desses atributos. Para isso é somente necessário que o preceptor tenha o certificado de conclusão da RM, o que lhe confere o Título de Especialista, pelo MEC. Muito é exigido do preceptor: conhecimento, sensibilidade, bom-senso, criatividade, capacidade de improvisação e compreensão da dinâmica ensino-aprendizagem ${ }^{16,17}$, mas não existe, por parte da CNRM, nenhum programa para qualificação, capacitação, formação pedagógica e didática, nem remuneração para estes profissionais, como se para ser preceptor bastasse ser apenas um "bom médico"18.

O desenvolvimento de habilidades técnicas é o marco fundamental da RM em CG e, para isso, é preciso um preceptor capacitado, com conhecimento técnico amplo, profundo, capacidade de transmissão de conhecimentos e de provocar no aprendiz o ganho de outras competências, como ética e humanismo. Ninguém nasce preceptor. Paulo Freire diz que "ninguém começa a ser educador numa certa terça-feira às quatro horas da tarde... A gente se faz educador na prática e na reflexão sobre a prática" 19 Embora alguns já tragam em sí esta tendência, a preceptoria se desenvolve e se aperfeiçoa por meio da reflexão sobre o exercício do trabalho e sobre o papel na formação do futuro profissional médico, implica em rever, repensar, reeducar sua própria prática. Segundo Blue ${ }^{20}$ uma preceptoria de qualidade é capaz de influenciar positivamente a performance do aprendiz.

Até agora, na formação em saúde, o domínio cognitivo tem sido privilegiado. É necessário um comportamento ativo do preceptor no sentido de que sejam desenvolvidos os saberes igualmente em suas dimensões profundas para que seja possível oferecer ao paciente uma atenção integral humanizada.

A RM é considerada o melhor método de treinamento após a graduação. É baseada no treinamento em serviço ${ }^{9}$. Desde que William Halsted ${ }^{21}$ a implantou em 1889, nos Estados Unidos da América, cirurgiões têm sido treinados no mesmo processo de formação. Sempre houve um "entendimento tácito" de que os cirurgiões são capazes de exercer essa função. Nunca se verificou se os responsáveis pelo treinamento, no mais importante dos cenários da prática da residência em CG, o Centro Cirúrgico, podem, de fato e de direito, se apossar do título "preceptor".

A figura central do cuidado é o paciente, motivo pelo qual o treinamento em cirurgia deve partir do princí- pio mais importante: sua segurança. Por isso a preceptoria no CC em todo o treinamento, durante todos os momentos da operação é mandatória. O preceptor deve assumir vários papéis nesse processo. Algumas vezes, mostra o caminho, serve como guia, outras vezes estimula o raciocínio e a postura ativa do residente, estimulando também sua capacidade de liderança da equipe. Aconselha, usando sua experiência, cuidando do crescimento profissional e pessoal do seu residente, na perfeita interação que o conceito de preceptor tem com o de orientador, supervisor, tutor e mentor ${ }^{2}$.

Pesquisa recente mostra ${ }^{5}$ que estudantes e jovens médicos identificam entusiasmo, compaixão, abertura, integridade e bom relacionamento com os pacientes, como atributos positivos a serem copiados de seus modelos. Características como entusiasmo, organização, envolvimento, além do conhecimento, estão entre as características necessárias aos preceptores ${ }^{20}$. Os aprendizes são atraídos por figuras importantes que incorporam responsabilidade e posição social. Alguns cirurgiões mais experientes mostram atitudes pouco desejáveis e um comportamento antiético, causando confusão, angústia e decepção em jovens médicos e estudantes sob sua supervisão22. O comportamento profissional e a ética devem ser explicitamente ensinados, estimulados, através de discussão franca entre preceptores e aprendizes ${ }^{5}$.

Existe um entendimento não escrito que os docentes são "preceptores naturais", como se o simples fato de ser aprovado em concurso para a docência os capacitasse para tal. Ao mesmo tempo, existe um certo (pre)conceito de que nos HNU não há profissionais habilitados para o exercício da preceptoria. Para que as dificuldades desse exercício, em qualquer tipo de hospital, sejam minimizadas é preciso que preceptores desenvolvam reflexão sobre sua prática, e que ensinem seus aprendizes a ocupar-se do ser humano, singular, inserido em um contexto social em constante transformação. Numa época em que se percebe o individualismo como comportamento habitual, a vida muitas vezes é relegada ao descuido. É também função do preceptor favorecer a união da realidade social e a experiência individual para o desenvolvimento de uma boa relação médico-paciente.

O produto da educação de profissionais de saúde deve ser um profissional que, além de excelente técnico, também seja dotado de traços humanísticos ${ }^{23}$. A capacitação de novas gerações de cirurgiões é tarefa complexa e de alta prioridade. Esse treinamento, na RM deve objetivar a aprendizagem real e integrada de todos os saberes, para que se formem profissionais moralmente confiáveis e disciplinados, além de excelentes técnicos. Os estudos de Blue ${ }^{20}$ concluíram que os aprendizes expostos ao convívio com preceptores ruins tiveram piores resultados em testes de avaliação cognitiva.

Há, no momento, dúvidas quanto às formas para esse treinamento ${ }^{22}$. Faz parte do bom profissional, cultivar 
a educação permanente, onde se aprende a lidar com os problemas do cotidiano do trabalho, considerando as experiências e as vivências de cada um, e, a partir dessa reflexão, promover transformações em sua prática profissiona. Esse aprimoramento deve ser constante ao longo da carreira profissional do médico, independentemente do tipo de hospital onde atua.

Para os jovens cirurgiões em formação, de fundamental importância são as observações, correções e incentivos (feedback). O preceptor deve usar sua própria bagagem intelectual e experiência e, por meio da observação direta, de uma atitude madura e reflexiva, detectar erros, mostrar como corrigi-los e estimular o aprendiz a busca ativa do conhecimento e da perfeição técnica.

Concluindo, os dois programas apresentaram preceptoria em níveis semelhantes, embora haja divergências no quesito "fica concentrado na operação". No entanto, para que esta conclusão seja amplamente aceita, é preciso uma comparação com um número maior programas de residência médica.

\title{
A B S T T R A C T
}

\begin{abstract}
Background: Medical residency is well known as the best training method after graduation. It is a moment when, in addition to receiving technical guidance, residents should also develop attitudes, ethics, and professionalism. In order for that to occur, preceptors should be prepared for their task. Objective: To evaluate and compare the preceptorship in the Medical Residency in General Surgery program, in the operating room of a Teaching Hospital (TH) and a Non-teaching Hospital (NTH), from the viewpoint of the residents who entered in 2010 and 2011. Methods: A questionnaire was applied to the residents, adapted from Sarker, Vincent and Darzi, and the Likert scale was used to qualify the survey items on the preceptors' attitudes. Results: At the TH, 12 preceptors were evaluated by 7 residents. One of the residents did not answer the questionnaire. At the NTH, 11 preceptors were evaluated by 13 residents. The comparison of the distribution of responses between hospitals was analyzed using the chi-squared test for trend. Significance level was set at 5\%. The statistical analysis was processed by the statistical software SAS ${ }^{\circledR}$ System version 6.11 (SAS Institute, Inc., Cary, North Carolina). The NTH showed a greater trend for discordant and indifferent responses than the TH. Only one question resulted in statistical significance between the hospitals. No significant difference was found between hospitals in the comparison of the responses to the remaining Conclusion: Only question 4 ("Keeps focused on the operation") showed a significant difference $(\mathrm{p}=0.010)$ in the distribution of responses. The two hospitals exhibited similar preceptorship quality.
\end{abstract}

Key words: Evaluation. Education, professional. Internship and residency. Preceptorship. General surgery.

\section{REFERENNCIAS}

1. Botti SHO, Rego STA. Docente-clínico: o complexo papel do preceptor na residência médica. Physis (RJ). 2011;21(1):65-85.

2. Botti SHO. O papel do preceptor na formação de médicos residentes: um estudo de residências em especialidades clínicas de um hospital de ensino [tese]. Rio de Janeiro: Fiocruz, Escola Nacional de Saúde Pública Sérgio Arouca; 2009.

3. Martins JC. Vygotsky e o papel das interações sociais na sala de aula: reconhecer e desvendar o mundo. Série Ideias [online]. 1997 [Acessado em set. 2011]; 28:111-22. Disponível em: http:// www.crmariocovas.sp.gov.br/dea_a.php?t=002

4. Leite LB. As dimensões interacionista e construtivista em Vygotsky e Piaget. Cadernos Cedes. Campinas: Papirus; 1991

5. Paice $E$, Heard $S$, Moss F. How important are role models in making good doctors? BMJ. 2002;325(7366):707-10

6. Perrenoud P. Construindo competências. In: Nova Escola (Brasil) [online]. 2000 [Acessado em jul. 2011].1:19-31. Disponível em: http://www.unige.ch/fapse/SSE/teachers/perrenoud/php_main/ php_2000/2000_31.html.

7. Silva Filho HP. O empresariado e a educação. In: Ferretti JC, organizador. Novas tecnologias, trabalho e educação: um debate multidisciplinar. 3a ed. Petrópolis: Vozes; 1996. p. 87-92.

8. Ribeiro MAA. Apontamentos sobre a Residência Médica no Brasil [online]. Câmara do Deputados, Brasil: Biblioteca Digital da Câmara dos Deputados; 2011 [Acessado em nov. 2011]. Disponível em http://bd.camara.gov.br/bd/bitstream/handle/bdcamara/6065/ apontamnetos_residência_ribeiro.pdf?sequence=1.

9. Brasil. Ministério da Educação e Cultura. Residências em Saúde. Residência Médica. Decreto $n^{\circ} 80.281$, de 05 de setembro de 1977. Regulamenta a Residência Médica e cria a Comissão Nacio- nal de Residência Médica. Acessado em jul. 2011. Disponível em: http://portal.mec.gov.br/index.php?option=com_content\&view= article\&id $=12263 \&$ Itemid $=507$

10. Sarker SK, Vincent C, Darzi AW. Assessing the teaching of technical skills. Am J Surg. 2005;189(4):416-8.

11. Likert R. A technique for the measurement of attitudes. Arch Psychol. 1932;22(140):1-55.

12. Mills JE, Francis $\mathrm{KL}$, Bonner A. Mentoring, clinical supervision and preceptoring: clarifying the conceptual definitions for Australian rural nurses. A review of the literature. Rural Remote Health. 2005;5(3):410.

13. Hauge LS, Wanzek JA, Godellas C. The reliability of an instrument for identifying and quantifying surgeons' teaching in the operating room. Am J Surg. 2001;181(4):333-7.

14. Al-Rubaish AM, Abdel Rahim SI, Hassan A, Ali AA, Mokabel F, Hegazy $M$, et al. Developing questionnaires for students' evaluation of individual faculty's teaching skills: A Saudi Arabian pilot study. J Family Community Med. 2010;17(2):91-5.

15. Velmahos GC, Toutouzas KG, Sillin LF, Chan L, Clark RE, Theodorou $D$, et al. Cognitive task analysis for teaching technical skills in an inanimate surgical skills laboratory. Am J Surg. 2004;187(1):114-9.

16. Ribeiro VMB. Formação pedagógica de preceptores do ensino em saúde. Juiz de Fora: Universidade Federal de Juiz de Fora; 2011.

17. Wuillaume SM. O processo ensino-aprendizagem na residência médica em pediatria: uma análise [tese]. Rio de Janeiro: Fiocruz, Instituto Fernandes Figueira; 2000.

18. Nunes MPT, Michel JLM, Haddad AE, Brenelli SL, Oliveira RSB. A Residência Médica, a preceptoria, a supervisão e a coordenação. Cadernos da ABEM. 2004;1:35-40.

19. Paulo Freire apud Carabetta Júnior V. Rever, pensar e (re)significar: a importância da reflexão sobre a prática na profissão docente. Rev bras educ med. 2010;34(4):580-6. 
20. Blue AV, Griffith CH 3rd, Wilson J, Sloan DA, Schwartz RW. Surgical teaching quality makes a difference. Am J Surg. 1999;177(1):869.

21. William Halsted. Encyclopedia of World Biography. 2004. Acessado em ago. 2011 em Encyclopedia.com. Disponível em: http:// www.encyclopedia.com/doc/1G2-3404708031.html

22. Jesus LE. Ensinar cirurgia: como e para quem? Rev Col Bras Cir. 2008;35(2):136-40.

23. Cox M, Irby DM. Teaching surgical skills - changes in the wind. $N$ Eng J Med. 2006;355(21):2664-9.
Recebido em

Aceito para publicação em

Conflito de interesse: nenhum

Fonte de financiamento: nenhuma

\section{Como citar este artigo:}

Santos EG, Ferreira RR, Mannarino VL, Leher EMT, Goldwasser RS, Bravo Neto GP. Cirurgia Geral, no centro cirúrgico, comparação entre um hospital universitário e um hospital não universitário. Rev Col Bras Cir. [periódico na Internet] 2012; 39(6). Disponível em URL: http:// www.scielo.br/rcbc

Endereço para correspondência:

Elizabeth Gomes dos Santos

E-mail: eligsant54@gmail.com 